\title{
Evaluation of the effect of six different paint cross section preparation methods on the performances of Fourier Transformed Infrared microscopy in attenuated total reflection mode
}

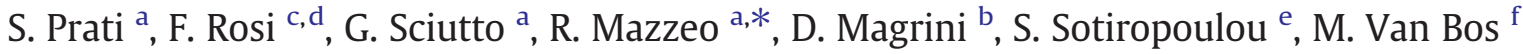 \\ a University of Bologna, Ravenna Campus, Microchemistry and Microscopy Art Diagnostic Laboratory (M2ADL), via Guaccimanni 42, 48100 Ravenna, Italy \\ b Institute for Conservation and Promotion of Cultural Heritage ICVBC-CNR, Sesto Fiorentino, Italy \\ c CNR-ISTM c/o Dipartimento di Chimica Università degli studi di Perugia, Italy \\ d Centro SMAArt c/o Dipartimento di Chimica Università degli studi di Perugia, Italy \\ e ORMYLIA Foundation, Art Diagnosis Centre, Omylia, Greece \\ ${ }^{\mathrm{f}}$ KIK-IRPA, Brussels, Belgium
}

\section{A R T I C L E I N F O}

\section{Article history:}

Received 22 December 2011

Received in revised form 16 January 2012

Accepted 16 January 2012

Available online 21 January 2012

\section{Keywords:}

Paint cross sections

FTIR microscopy in ATR mode

$\mathrm{KBr}$

Cyclododecane

Argon ion milling

\begin{abstract}
A B S T R A C T
The purpose of the present research was to compare the effect of different embedding and polishing procedures on the performances achievable with Fourier Transformed Infrared (FTIR) microscopy in Attenuated Total Reflection (ATR) mode. The attention was specifically focused on this technique since it is generally considered one of the most appropriate methods to analyze cross sections, thanks to the fact that the recorded spectra are similar to those collected in transmission mode.

The research work was structured as a round robin among six different conservation and research laboratories. They were asked to embed fragments derived from the same area of a real sample following each one a different procedure. The performances of FTIR microscopy in ATR mode were evaluated on the different cross sections, considering the cross section morphology and the possible source of contamination. The cross sections were previously observed with optical microscopy, Environmental Scanning Electron Microscope (ESEM) and confocal microscopy.

Three procedures were eventually selected, because they provided the best results in terms of both limiting the contamination of the embedding medium and achieving good contact with the ATR crystal. They were: a) embedding in $\mathrm{KBr}$; b) cyclododecane pre-treatment before embedding in an organic resin and cutting of the sample; c) embedding in an organic resin and polishing with argon ion milling.

These three procedures were finally compared and the weak points outlined, indicating where improvements could be made for further researches.
\end{abstract}

(c) 2012 Elsevier B.V. All rights reserved.

\section{Introduction}

The preparation of polychrome samples has become a crucial concern since the introduction of techniques able to analyze and localize at a micrometric-scale both organic and inorganic materials directly on thin or cross-sections [1-13].

The preparation of the sample stratigraphy in the form of thin or cross section is normally accomplished by embedding the paint fragment in a polymer resin, followed by grinding, polishing or microtoming. However, the penetration of the embedding resin (epoxy, polyester, acrylic, etc.) inside porous paint samples can negatively affect the characterization of the organic components, especially the uppermost varnish layer of a painting and its preparatory ground.

\footnotetext{
* Corresponding author.

E-mail address: rocco.mazzeo@unibo.it (R. Mazzeo).
}

Smearing of the resin over the whole section surface, during the polishing procedure, may also occur. When, for instance, the thin or cross section is analyzed by spectroscopic techniques, such as FTIR and Raman microscopy, the resin absorption bands may overlap those characteristic of the organic paint components. A correct methodological approach can prevent such limitations. For instance, the surface analysis of the sample, performed before embedding it for cross section preparation, is an alternative procedure for the characterization of preparatory grounds and external varnish layers [2].

So far, several embedding systems have been proposed to the aim of reducing the contamination of the embedding resin. Various barrier coatings have been tested to prevent its infiltration into the sample [7]. To this purpose the use of cyclododecane $\left(\mathrm{C}_{12} \mathrm{H}_{24}\right)$ was recently proposed as a temporary barrier [8]. Cyclododecane is ordinarily used in the conservation field mainly as a temporary consolidant or a hydrophobic barrier coating for aqueous treatments of watersensitive artworks, thanks to its property to sublimate at room 
temperature $[14,15]$. The sample preparation method proposed by de Fonjaudran et al. [8] is based on the consolidation of the porous paint sample, either with a saturated solution of cyclododecane in toluene $(80 \% \mathrm{w} / \mathrm{v})$, or with molten cyclododecane. The sample is subsequently embedded in resin and then cut by microtomy. The resulting cross section cannot be analysed earlier than $24 \mathrm{~h}$, as this is the time needed by cyclododecane to sublimate [8]. Even though such a method limits interferences from embedding materials, the authors give account of various drawbacks, such as the significant sample manipulation required, which may not be possible for very small samples, and the potential reduction of the cross section stability after the sublimation of the cyclododecane. Moreover, the obtained samples appear to be less photogenic when compared with traditional sample preparation methods [8].

The use of infrared transparent salts as cross section embedding materials was introduced to the same purpose of avoiding contamination effects from the embedding resin and improving the detection of organic substances. In particular, potassium bromide ( $\mathrm{KBr})$, commonly employed in the preparation of transmission pellets, resulted very promising. As previously demonstrated [2], the procedure to obtain a cross section is rather simple and the detection of organic substances can be therefore improved. In addition, the $\mathrm{KBr}$ embedding system leads also to better photographic optical microscopy documentation of paint cross sections when observed under ultraviolet illumination, thanks to the absence of the contribution of the embedding resin fluorescence. However, the polishing procedure is still a crucial matter, since $\mathrm{KBr}$ is hygroscopic and more delicate than an organic resin material. The spectral quality is therefore reduced during the acquisition (particularly after the first 20 min of FTIR spectra acquisition). Other less hygroscopic infrared salts have been investigated, such as calcium fluoride $\left(\mathrm{CaF}_{2}\right)$ and barium fluoride $\left(\mathrm{BaF}_{2}\right)$, but the advantage of their lower hygroscopicity does not outweigh the disadvantages of the physical and mechanical properties of the obtained cross sections $\left(\mathrm{CaF}_{2}\right.$ cross sections are not transparent; $\mathrm{BaF}_{2}$ cross sections are more fragile than $\mathrm{KBr}$ ones) [10].

Over the last years specific researches have been devoted to the study of preparation methods for thin sections to be subsequently analysed in transmission mode [3-6]. Various strategies have been proposed, employing either infrared inactive salts [3], aluminium foil protections of the sample before embedding [5], analyses of fragments containing the complete stratigraphy after their compression with a micro anvil cell [5], or of non-embedded microtomed samples [6].

Samples prepared as thin sections provided better results, compared with cross sections, when submitted to FTIR microscopy, Micro-X-Ray Diffraction ( $\mu$ XRD), Micro-X-Ray Absorption Near Edge Spectroscopy ( $\mu$ XANES) analyses in transmission mode [5]. Nevertheless, the thin section preparation is more destructive and still more critical than cross section preparation.

FTIR microscopy in ATR mode is generally considered to be one of the most appropriate methods to analyze cross sections, thanks to the fact that the recorded spectra result to be similar to those collected in transmission mode [16,17]. Moreover, thanks to the magnification factor of the Internal Reflection Element (IRE), the ATR configuration allows smaller areas to be investigated when compared with reflection or transmission mode $[18,19]$. The dimension of the investigated area in a microscope can be estimated with the Rayleigh criterion:

$d=1.22 \lambda / n_{1} \sin \theta$

where $d$ is the spatial resolution, $\lambda$ is the wavelength, $n_{1}$ is the refractive index of the medium in which the measurement is conducted (i.e. the IRE or crystal), and $\theta$ is the most extreme ray entering the objective [19]. As an advantage a better spatial resolution can be achieved maintaining a good spectral quality, while drawbacks of this method are the possible denting caused by the crystal on the cross section surface and the necessity of a uniform contact between the sample and the ATR crystal, implying an accurate surface preparation $[18,20]$.

Linear array detectors (raster scanning) have been developed in recent years, combining together several mercury cadmium telluride (MCT) detectors with a motorized stage to perform sequential scan lines. This system has an acquisition time reduced by a factor corresponding to the number of detector elements. The individual elements are $25 \mu \mathrm{m}$ in size and this allows to obtain spectra with a good spectral quality [21]. In transmission or reflection mode, the achieved resolution of $25 \mu \mathrm{m}$ can be reduced to $6.25 \mu \mathrm{m}$ using an optical zoom. In ATR mode, the spatial resolution is $\sim 6 \mu \mathrm{m}$ when a germanium crystal is used as IRE.

Although it is known that the roughness of the surface is crucial to optimise the ATR crystal/sample surface, scarce attention has been devoted to evaluate the polishing effects on the surface morphology. Some studies have already been performed by employing Argon Ion Milling or controlled sample polishing systems [12,13] to obtain flat surfaces to be analyzed by FTIR microscopy in total reflection mode, even though a well standardised and repeatable method has not yet been developed and the effects of such procedures on the results achievable by means of FTIR microscopy in ATR mode have not been studied.

Two main problems related to the sample morphology may occur when micro ATR analyses are performed on paint cross section:

- Inadequate contact between crystal and sample

- The analyzed sample area may vary from the one originally selected, due to slipping of the sample when the crystal comes in contact with it.

Considering the crystal dimension of the ATR used in the present research, the contact surface between the sample and the crystal is around $300 \mu \mathrm{m}$, even though the analysed area can be only about $20 \mu \mathrm{m}$, depending on the employed aperture. The morphology of the sample surface is a crucial factor because it strongly affects the quality of the crystal/sample contact and consequently the spectral signal. On the other side, a movement of the sample may occur

Table 1

Embedding and polishing procedures.

\begin{tabular}{|c|c|c|}
\hline Sample name & Embedding procedures & Polishing procedures \\
\hline ROND13_1 & Polyester bicomponent Mecaprex 2S Presi, & $\begin{array}{l}\text { Buehler silicon carbide for wet grinding using different grit sizes (120-400-600-1200 mesh) } \\
\text { and final polishing with chamois clothes }\end{array}$ \\
\hline ROND13_2 & $\begin{array}{l}\text { PVA to fix the sample } \\
\text { Embedding Resin :polymethyll methacrylate Spofacryl } \\
\text { Cube: Plexiglas (PMMA) bar }\end{array}$ & $\begin{array}{l}\text { Struers silicon carbide papers for wet grinding using different grit sizes } \\
(120-180-220-320-500800-1200-2400-4000)\end{array}$ \\
\hline ROND13_3 & $\begin{array}{l}\text { Coating the sample with } \mathrm{C}_{12} \mathrm{H}_{24} \text { (from Zecchi) Embedding the } \\
\text { sample in epoxy resin Cristal EP Bresciani srl from Milano }\end{array}$ & Removal of resin excess by cutting (no polishing procedures was performed) \\
\hline ROND13_4 & Polyester resin (Alec Tiranti Ltd.) & Argon ion milling (12 h, $5 \mathrm{Kv})$ \\
\hline ROND13_5 & $\begin{array}{l}\text { KBr pellet: Fluka } 34919 \\
\text { Spectranal grade }\end{array}$ & $\begin{array}{l}\text { Dry polishing with Struers Waterproof Silicon Carbide Paper P 500, 800, } 1000 \\
\text { and Micromesh Carbide Paper 4000, 6000, 8000, } 12,000\end{array}$ \\
\hline ROND13_6 & $\begin{array}{l}\text { KBr pellet Fluka } 60095 \\
\text { Crystallised, purity }>=99.0 \%\end{array}$ & Dry polishing with Struers Waterproof Silicon Carbide Paper P \# 1000, 1200, 2500, 4000 \\
\hline
\end{tabular}




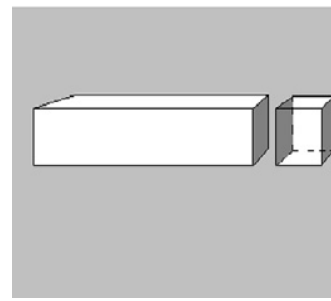

A

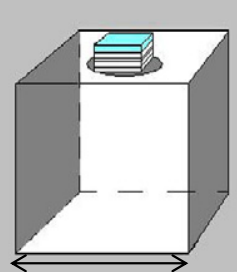

B

$1 \mathrm{~cm}$

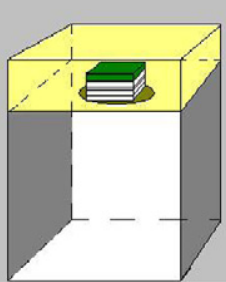

C

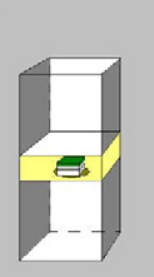

D

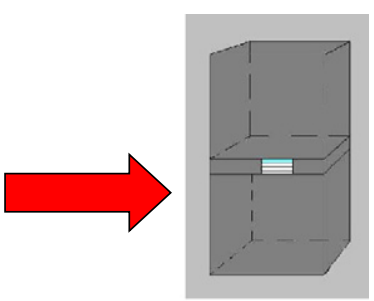

$\mathbf{E}$

Fig. 1. Scheme of ROND13_2 embedding procedure.

when the crystal comes in contact with an irregular surface, with areas at different levels, with soft materials or with materials of varying hardness. This movement can be of several microns, causing an incorrect registration of the analyzed area.

The aim of this paper is to evaluate the effect of sample preparation on the analytical performances of micro ATR by taking into account the surface morphology resulting from the adopted polishing procedure and the extent of sample contamination caused by the embedding material. To this purposes, and within the framework of the CHARISMA European project [Cultural Heritage Advanced Research Infrastructures: Synergy for a Multidisciplinary www.charismaproject.eu], a round robin was conducted on a real paint sample collected from a panel painting attributed to Baldassarre Carrari (1460-1516). The sample was subdivided into six smaller fragments, one for each of the six scientific laboratories involved in the project. The different cross section preparation and polishing methods applied by the different laboratories were then tested and the samples analyzed with confocal microscopy, optical microscopy, ESEM-EDX (Environmental Scanning electron microscope and energy dispersive X-ray microanalysis) and FTIR microscopy in ATR mode.

\section{Experimental}

\subsection{Materials}

Table 1 lists the different embedding and polishing procedures applied by each of the six laboratories on the different fragments originally derived from the same real sample (ROND13), which was collected from a panel painting attributed to Baldassarre Carrari (1460-1516). In more detail:

- ROND13_1 was simply embedded in a polyester bi-component resin and then polished.

- ROND13_2 was prepared following one of the procedures routinely in use at the KIK IRPA institute (Fig. 1), consisting of: sawing a Plexiglas bar into $1 \mathrm{~cm}^{3}$ cubes (A); fixing the sample onto one of the cubes with a drop of polyvinyl acetate glue (B); applying a small quantity of resin(C); positioning, after a few minutes, a second cube on top of the resin (D); finally polishing the obtained cross-section (E).

- ROND13_3 was prepared by immersing the fragment in melted cyclododecane $\mathrm{C}_{12} \mathrm{H}_{24}$ (CDD), then rapidly pulling it out and embedding it in epoxy resin. The sample stratigraphy was obtained

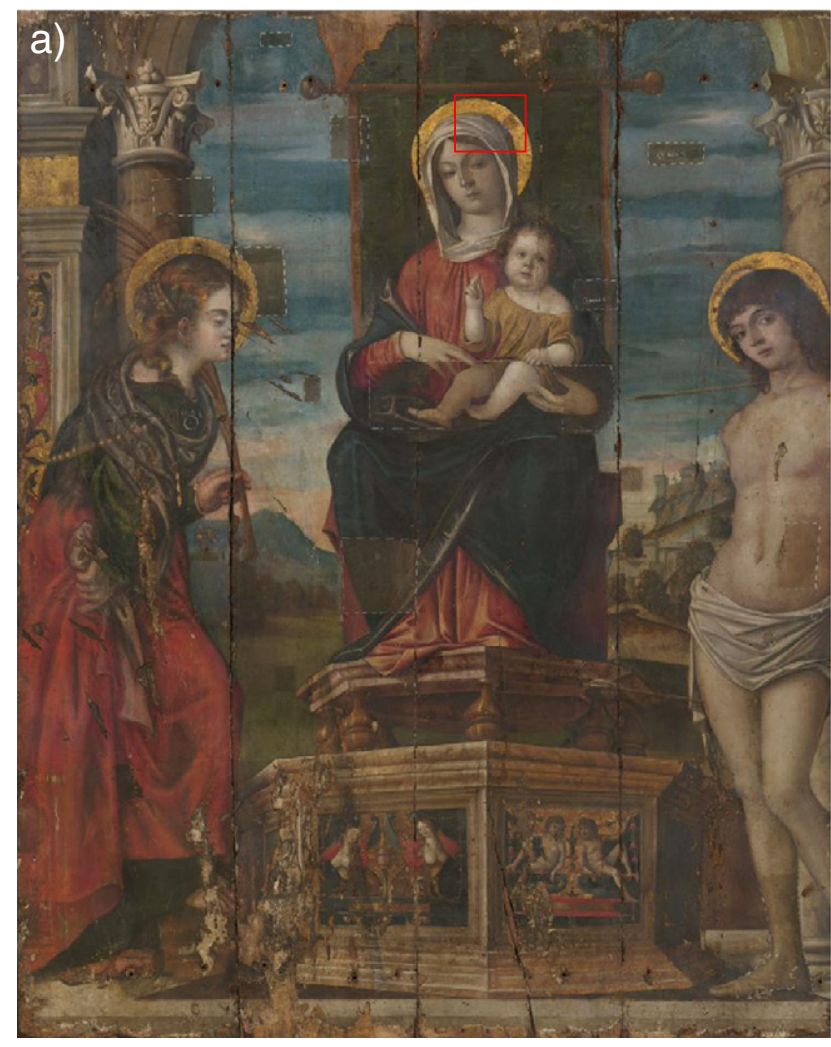

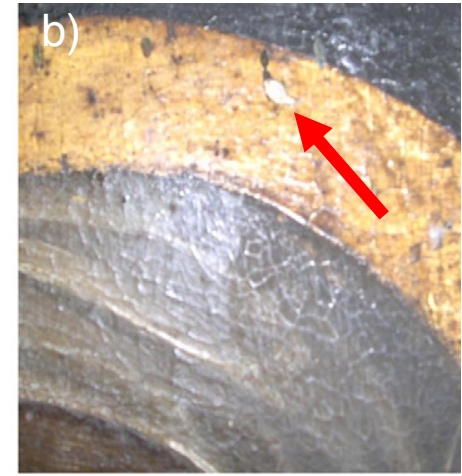

C)

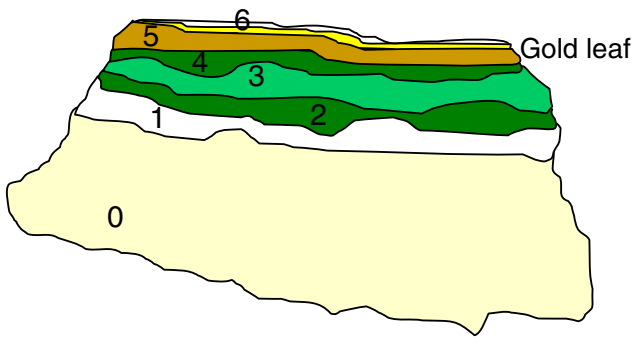

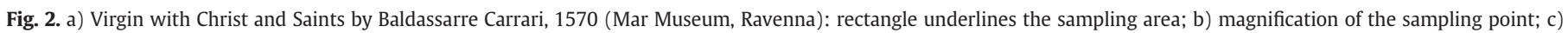
cross section scheme, numbers refer to Table 2 . 
Table 2

Description of the layers in ROND13.

\begin{tabular}{|c|c|c|c|c|}
\hline Layer n. & Thickness $(\mu \mathrm{m})$ & Colour & Composition & Band used for mapping $\left(\mathrm{cm}^{-1}\right)$ \\
\hline \multirow[t]{3}{*}{6} & & Brown & Calcium oxalate & 1321 \\
\hline & & & Silicate & Not mapped \\
\hline & $2-3$ & Gold leaf & & \\
\hline \multirow[t]{2}{*}{5} & $10-15$ & Beige-brown (mordant for the gold leaf) & Clay type earth pigment & 911 \\
\hline & & & Lipidic binder & 1740 (ester), 1710 (acid) \\
\hline \multirow[t]{3}{*}{4} & 15 & Dark green & Copper acetate-resinate & 1602 \\
\hline & & & Lead acetate or organo copper complex & 1550 \\
\hline & & & Lipidic binder & 1740 (ester), 1710 (acid) \\
\hline \multirow[t]{5}{*}{3} & $15-30$ & Light green & Copper acetate-resinate & 1602 \\
\hline & & & Lead acetate or organo copper complex & 1550 \\
\hline & & & Lipidic binder & 1740 (ester), 1710 (acid) \\
\hline & & & Copper oxalate & 1360 \\
\hline & & & Not identified compound & 3570,1351 \\
\hline \multirow[t]{4}{*}{2} & $8-10$ & Dark green & Copper acetate-resinate & 1602 \\
\hline & & & Lead acetate or organo copper complex & 1550 \\
\hline & & & Lipidic binder & 1740 (ester), 1710 (acid) \\
\hline & & & Calcite & 870 \\
\hline \multirow[t]{3}{*}{1} & 30 & White grey & Lead white & 837 \\
\hline & & & Lead carboxylates & Not mapped \\
\hline & & & Lipidic binder & 1740 (ester), 1710 (acid) \\
\hline 0 & 400 & Brownish white & Gypsum & Correlation map with standard gypsum \\
\hline
\end{tabular}

by cutting the hardened block with a diamond saw in wet condition. No polishing procedure was carried out.

- ROND13_4 was polished by means of argon ion milling, employing an IB-09010 JEOL cross section polisher (CP) with ultra pure argon (99.9999\%, purity grade 6.0). The instrument created a cross-section by irradiating the sample with an ion beam along the edges of the shield placed on it in the dry-evacuated chamber.

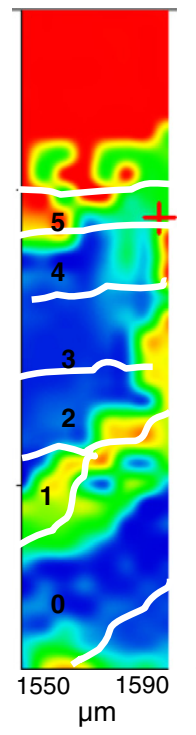

a) ROND13_1 Correlation map with the embedding resin

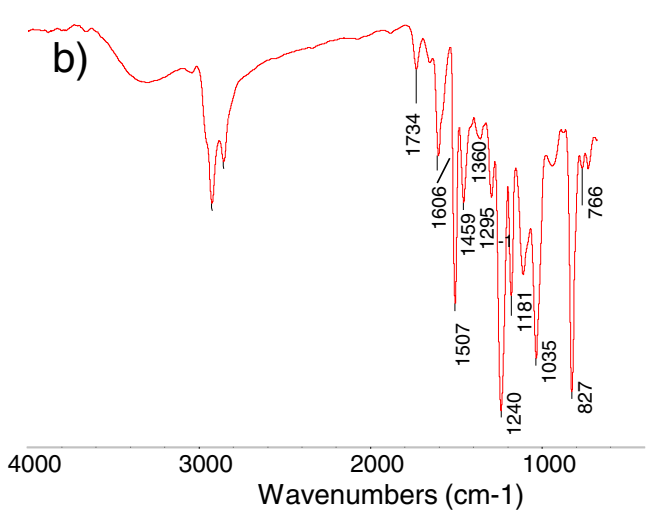

C) ROND13_2 Map profile $753 \mathrm{~cm}^{-1}$

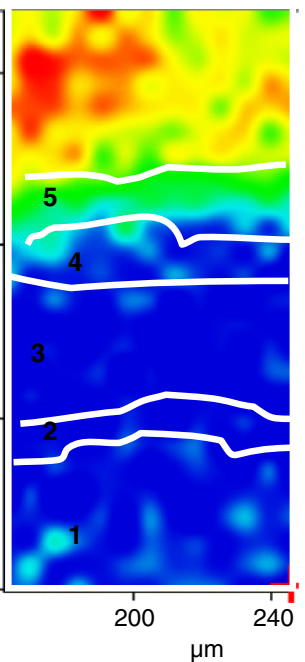

d)

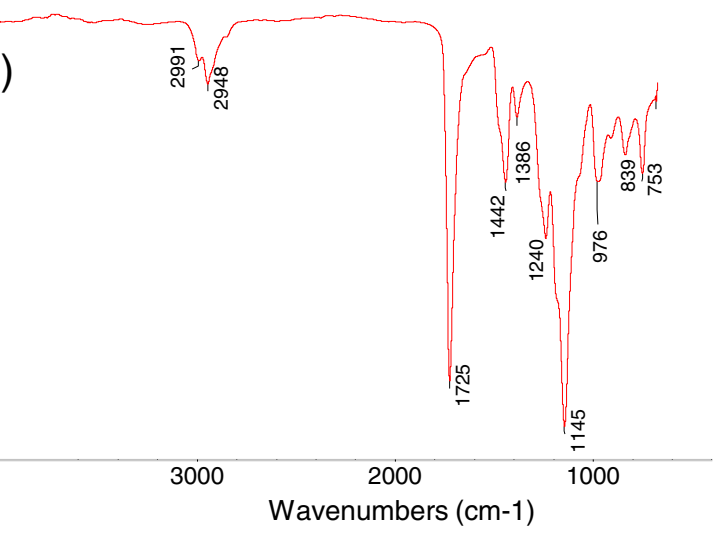

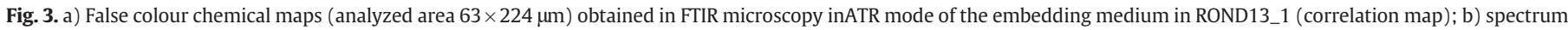

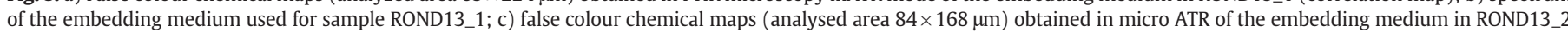
(map profile for peak $753 \mathrm{~cm}^{-1}$ ); d) spectrum of the embedding medium used for ROND13_2. 


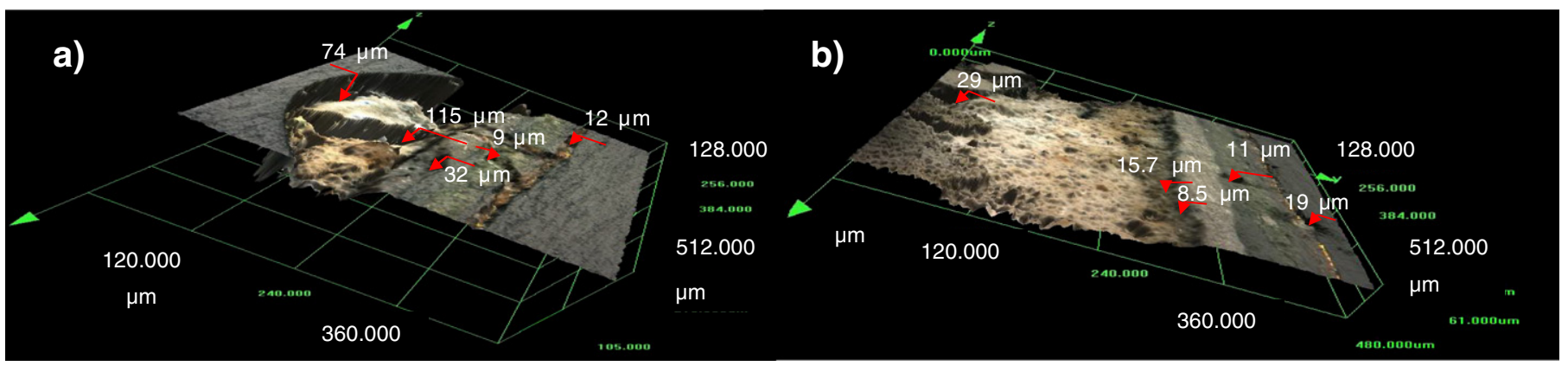

Fig. 4. Confocal microscopy images of: a) 3D, ROND13_1; b) 3D, ROND13_2.

- ROND13_5 and ROND13_6 were both $\mathrm{KBr}$ embedded samples, prepared following already reported procedures [2]. The polishing step was performed by two different operators, using different maximum grit size for the grinding paper.

Fig. 2 shows the area of the painting where ROND13 was collected from and the cross section scheme, while Table 2 summarizes the composition of each layer as detected by FTIR microscopy in ATR mode.
ROND13, collected from the Virgin's halo, resulted stratigraphically composed of a gypsum preparatory ground (layer 0 ), probably with a glue binder, over which a layer of lead white mixed with a lipidic binder had been applied (layer 1 ). This layer could be associated to the presence of an underpainted figure representing a saint, whose presence was later revealed by X Ray Radiography examinations. Three green paint layers with different tonalities (2-4) were present above the white layer and could be associated with the green hanging which extends beneath the Virgin's gold halo. They

\section{a)}

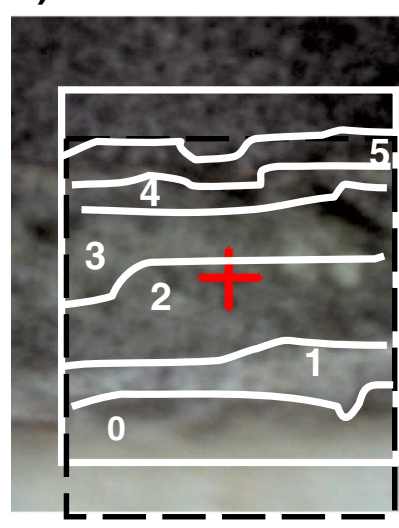

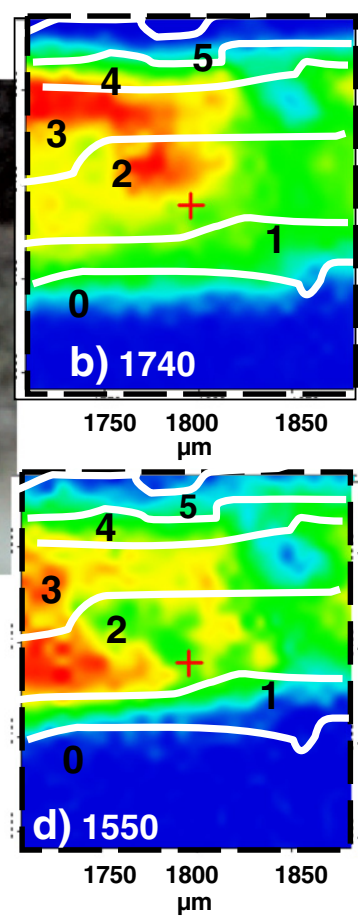
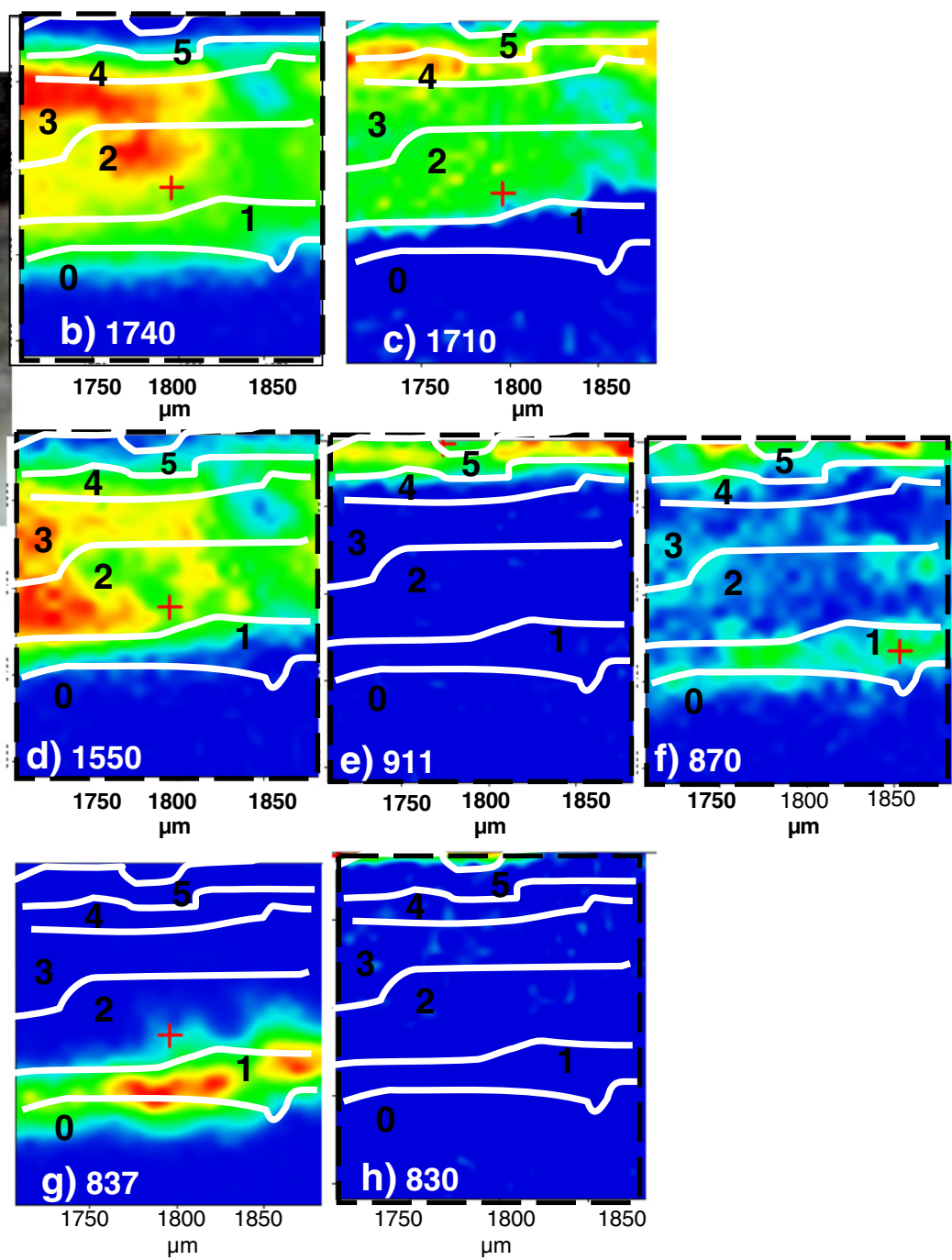

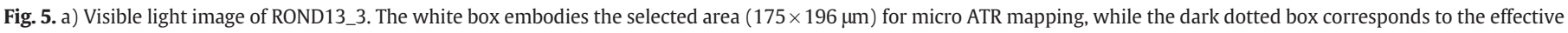

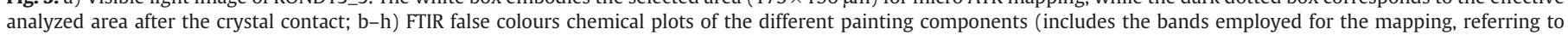
Table 2; band at $830 \mathrm{~cm}^{-1}$ was employed to map the embedding epoxy resin). 


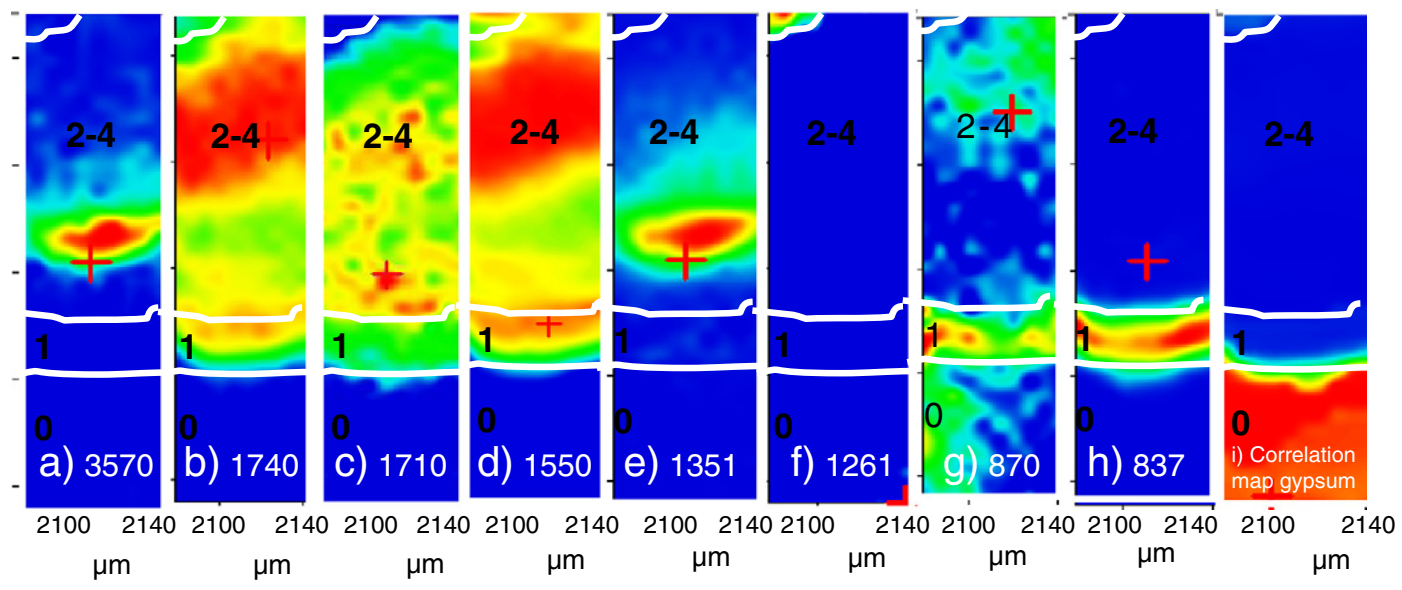

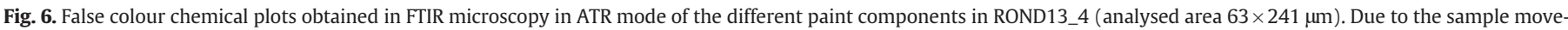

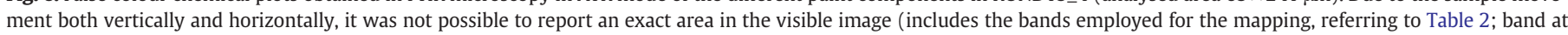
$1261 \mathrm{~cm}^{-1}$ was employed to map the embedding polyester resin).

resulted composed of copper acetate (verdigris) or copper resinate in an oily binder. Layer 3 was further characterised by the contemporary presence of lead white pigment particles. The gilding of the Virgin's halo had been achieved by applying a gold leaf over a layer of a clay type earth pigment in oil (layer 5). The paint stratigraphy revealed also the presence of a furthermore outermost varnish layer (layer 6).

\subsection{Analytical techniques}

- Dark field observations were performed using an Olympus BX51M optical microscope equipped with fixed oculars (magnification $10 \times)$ and objectives with different magnifications (5, 10, 20, 50 and $100 \times)$. Visible and ultraviolet light were respectively provided by a $100 \mathrm{~W}$ halogen projection lamp and an Ushio Electric USH102D lamp. Cross section photomicrographs were recorded by an Olympus DP70 digital scanner directly connected to the microscope.

- The documentation of sample surface morphology was carried out employing a confocal LEXT microscope purchased from Olympus. A $408 \mathrm{~nm}$ laser diode was combined with optics specifically designed for operations at this wavelength. 2D and 3D colour images were acquired at different magnifications $(5,10,20$, and $50 \times$ ) by a combination of laser image with full colour bright field image in the computer system.

- A Nicolet iN ${ }^{\mathrm{TM}} 10 \mathrm{MX}$ raster scanning microscope, fitted with a MCT detector cooled by liquid nitrogen, was employed to perform ATR mapping. The iN ${ }^{\mathrm{TM}} 10 \mathrm{MX}$ microscope has the peculiarity of integrating the interferometer, source, laser and detector into a single compartment, which yields a high energy output and a very high $\mathrm{S} / \mathrm{N}$ ratio. The mapping measurements were performed in ATR mode using a slide-on ATR objective with a conical germanium crystal, in the range $4000-680 \mathrm{~cm}^{-1}$, at a spectral resolution of $4 \mathrm{~cm}^{-1}$ and with 32 scans. The selected areas were analyzed with a step size of $7 \mu \mathrm{m}$ and an aperture of $60 \mu \mathrm{m} \times 60 \mu \mathrm{m}$ leading to a spatial resolution of about $15 \mu \mathrm{m}$.

\section{Discussion}

It is important to underline that this research was not aimed at studying the comprehensive composition of the selected sample, but at evaluating the effects the various sample preparation methods have on the results achievable through FTIR microscopy in ATR mode. To this purpose, the complexity and variability of the sample paint structure and composition represented an optimum bench test. As expected, the analyses performed in micro ATR mode showed that the achievable results can be seriously influenced by the adopted sample preparation method.

In particular, in ROND13_1 and ROND13_2 the false colour correlation maps of the polyester and acrylic embedding resins (Fig. 3) clearly showed that both samples were affected by infiltration and/ or spreading of the resin caused by the polishing procedure, even though ROND13_2 appeared to be less contaminated. Moreover, when ATR mapping of the ester and carboxylic acid bands was performed on both cross sectioned samples, the distribution of the organic paint binding media could not be distinguished from that of the embedding resin.

Another drawback of these two embedding procedures is the use of water as lubricant during polishing. This effect is particularly striking in the presence of water soluble materials, such as gypsum preparatory grounds. As a matter of fact, 3D confocal microscopy images (Fig. 4a-b) of ROND13_1 and ROND13_2 showed, after wet polishing, an irregular surface particularly localised in the preparatory ground
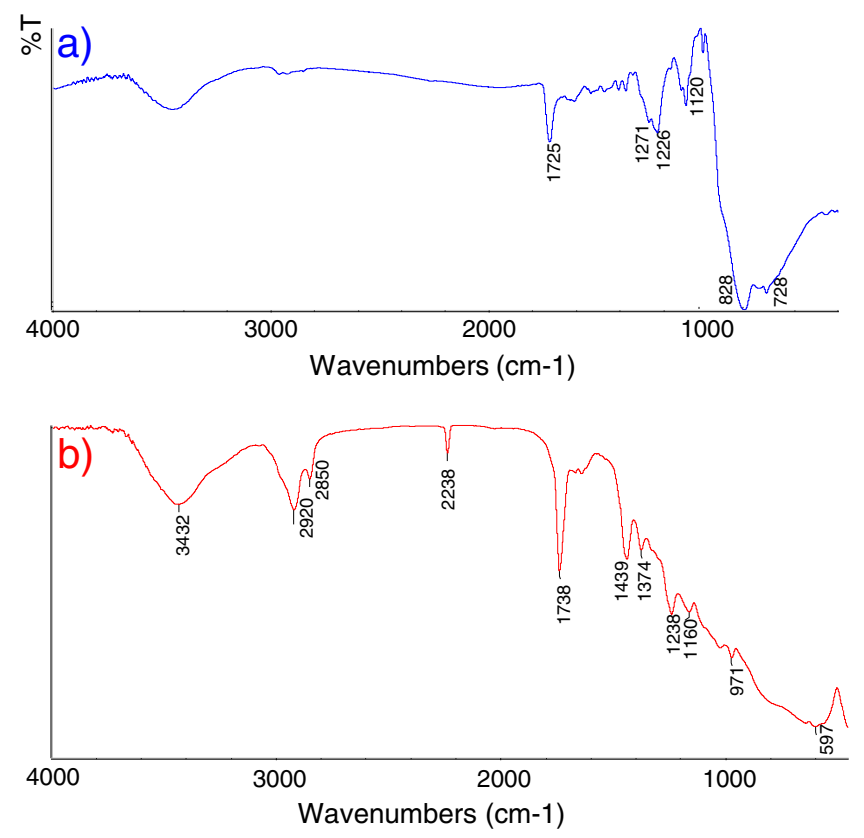

Fig. 7. IR spectra registered in transmission ( $\mathrm{KBr}$ pellet) on the abrasive surface sampled from: a) 4000 grit paper employed for polishing ROND13_6; b) 12000 grit paper employed for polishing ROND13_5. 
layer made of gypsum and glue, which were both dissolved by water during polishing. This can explain the reason why in both samples it was not possible to achieve any contact between the crystal and the preparatory ground layer. In ROND13_1, resulting more irregular than ROND13_2 as observed by confocal microscopy, no signal could be recorded, neither from layer 0 , nor from layer 1 . Cyclododecane (CDD), used for the preparation of ROND13_3, acted as a temporary barrier against the penetration of the embedding resin. Cyclododecane, after having exerted its barrier action, sublimated at room temperature within a few hours, leaving no trace of its previous presence. Moreover, the use of the cutting method prevented the spreading of the resin that is typical of the polishing procedure. Analyses performed in FTIR microscopy in ATR mode show that this treatment seems to be effective in reducing sample contamination from the embedding resin (Fig. 5). Indeed, the uppermost layer resulted to be the only one partially contaminated, as shown by the false colour chemical plot obtained for the band at $830 \mathrm{~cm}^{-1}$ used as a marker of the embedding epoxy resin (Fig. $5 \mathrm{~h}$ ).

In ROND13_4 the embedding resin was present only in a limited area, not affecting the sample characterisation (Fig. 6f).
As expected, no spectral interferences from the embedding medium were observed with $\mathrm{KBr}$ embedded samples, even though the dry polishing resulted in other sources of contamination. ROND13_6 proved to be contaminated by an organic compound characterised by spectral bands at $1725,1270,1225,1120,830 \mathrm{~cm}^{-1}$, present both on the sample surface and on the surrounding $\mathrm{KBr}$. In particular, the band at $1725 \mathrm{~cm}^{-1}$ prevented the identification of the organic paint substances. As confirmed by FTIR analyses (Fig. 7a), this contamination must be ascribed to the organic material present as binder of the SiC particles of the polishing abrasive papers. ROND13_6 was actually further polished with the finer papers used for ROND13_5, obtaining the removal of the contaminant from the cross section.

The abrasive papers employed for ROND13_5 were bound with another kind of polymer, probably containing an acrylonitrile component (bands at $2238 \mathrm{~cm}^{-1}$ in Fig. 7b). In spite of that, no traces of contamination were detected on the sample, probably because the polishing procedure had employed finer grind papers. Furthermore, ESEM-EDX surface analyses were able to detect $\mathrm{KBr}$ particles on ROND13_5. It cannot be excluded that intra layers contamination may arise from softer materials due to the dry polishing.
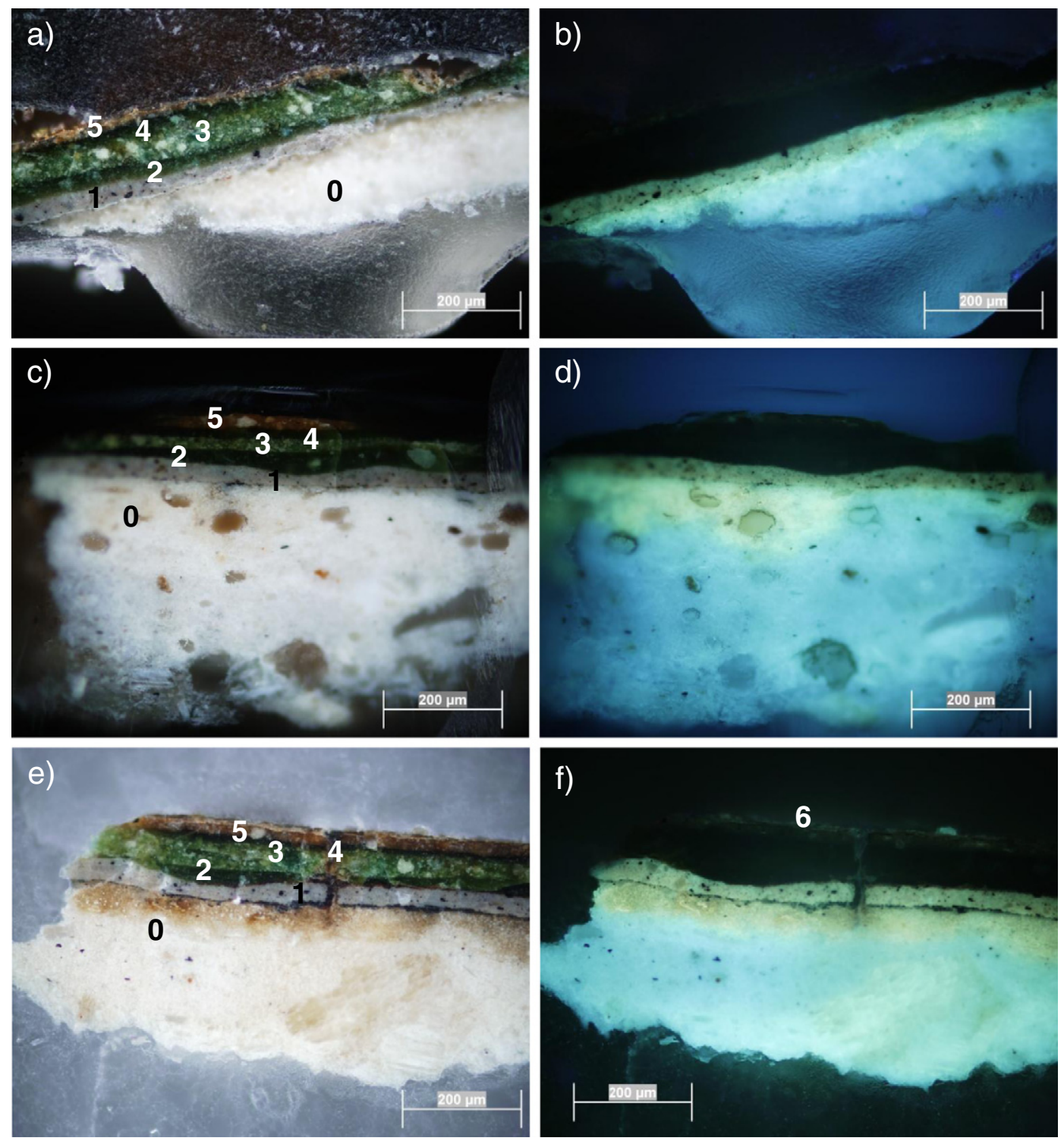

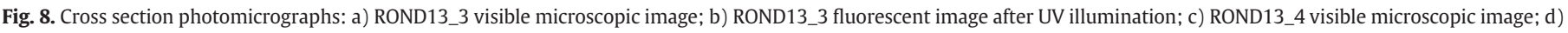

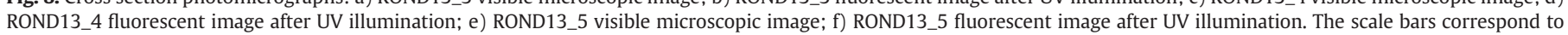
$200 \mu \mathrm{m}$. 
Since ROND13_1, ROND13_2 and ROND13_6 resulted to be contaminated by the embedding procedures, they were not further considered for the evaluation of the different degree of information achievable by ATR.

The spectral results from samples ROND13_3, ROND13_4, ROND13_5 were elaborated considering the inorganic and organic materials distribution within the differently prepared cross sections. Fig. 8 shows the cross section photomicrographs of the three samples under visible and UV illumination.

Starting from the gypsum ground layer, it was possible to clearly map its distribution both in ROND13_4 and in ROND13_5 (Figs. 6i, $9 \mathrm{~m})$. On the other hand, it was not possible to record spectra from the gypsum ground of wet polished ROND13_3. As with ROND13_1 and ROND13_2, confocal microscopy images acquired on ROND13_3 (Fig. 10a) evidently showed the negative role played by water in the partial removal of the gypsum ground. Compared to ROND13_1 and ROND13_2 (Fig. 4a-b), the absence of further polishing steps limited the solubilisation of the gypsum layer in ROND13_3, but generated an irregular surface caused by the different hardness of the materials (Fig. 10a). The irregular surface morphology prevented from a good ATR crystal contact, negatively affecting the spectral results.

In order to overcome such drawbacks, some tests are currently being carried out. They favour cyclododecane infiltration as consolidant, avoiding the solubilisation of water sensitive materials during the wet polishing or the cutting of the cross section.
ROND13_4 and ROND13_5 were obtained without any solvent but by ion milling (ROND13_4: Fig. 10b-c), or by dry polishing (ROND13_5: Fig. 10d,), obtaining in both cases a flat surface, which allowed the mapping of the gypsum preparation (Figs. 6i, 9m). For ROND13_4 (Fig. 10b-c), even though the surface seemed to be reasonably planar, the bidimensional confocal image showed a sort of curtaining effect due to differences in the etching rate, probably as a result of voids present in the sample [22].

It is worth mentioning that it was not possible to identify organic substances in the gypsum ground of any sample. It is well known that in Italy panels were traditionally treated for painting by the application of a preparatory ground made of gypsum and animal glue [23]. In this case the amount of a glue binder was probably lower than the instrument detection limit.

In all the three cross sections the lead white layer (layer 1) was easily mapped by integrating the bending mode of the carbonate anion at $837 \mathrm{~cm}^{-1}$ (Figs. 5g, 6h, 9l). Lead white had been applied in an oil binding medium, which was reliably mapped by integrating the ester group carbonyl band at $1740 \mathrm{~cm}^{-1}$ in all the samples (Figs. 5b, 6b, 9b). All the three samples showed, in this layer, the contemporary presence of acidic components and carboxylates, even though spectra acquired from ROND13_4 appeared to be noisier, probably because of a not optimal contact. Indeed, the signal associated to the acidic component in layer 1 was so low for ROND13_4 that it was not possible to map it (Fig. 5c).

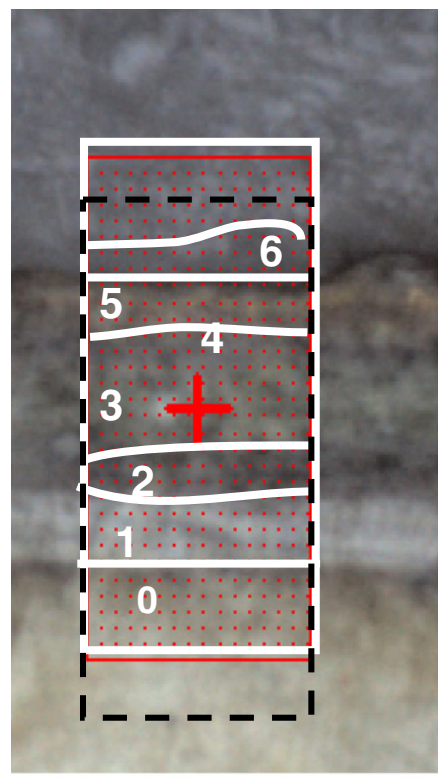

a)
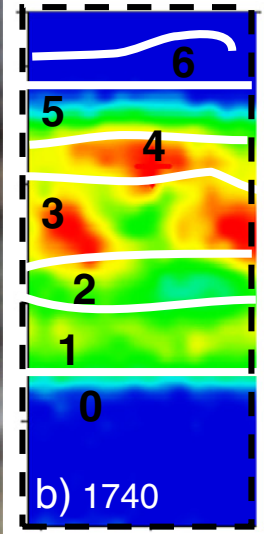

1800



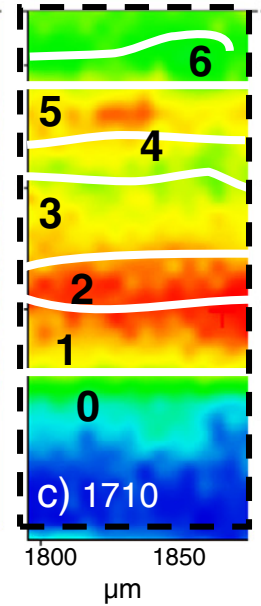

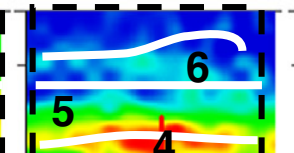

1

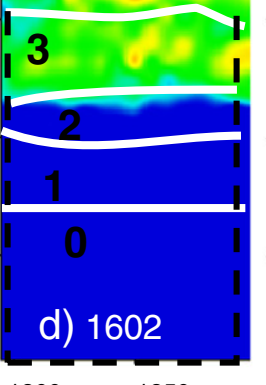

$1800 \quad 1850$ $\mu \mathrm{m}$
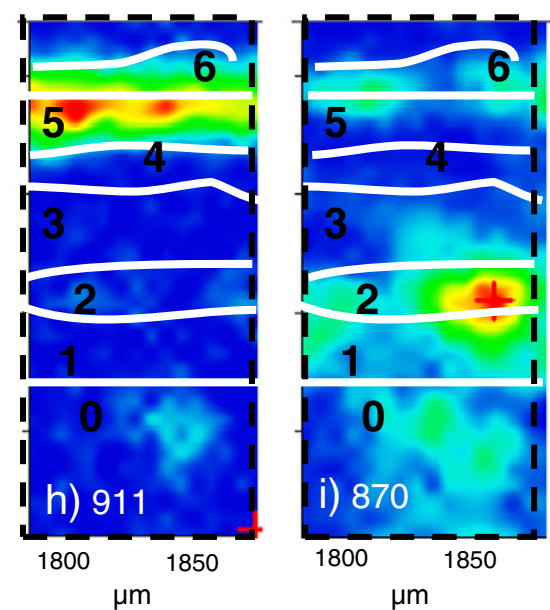
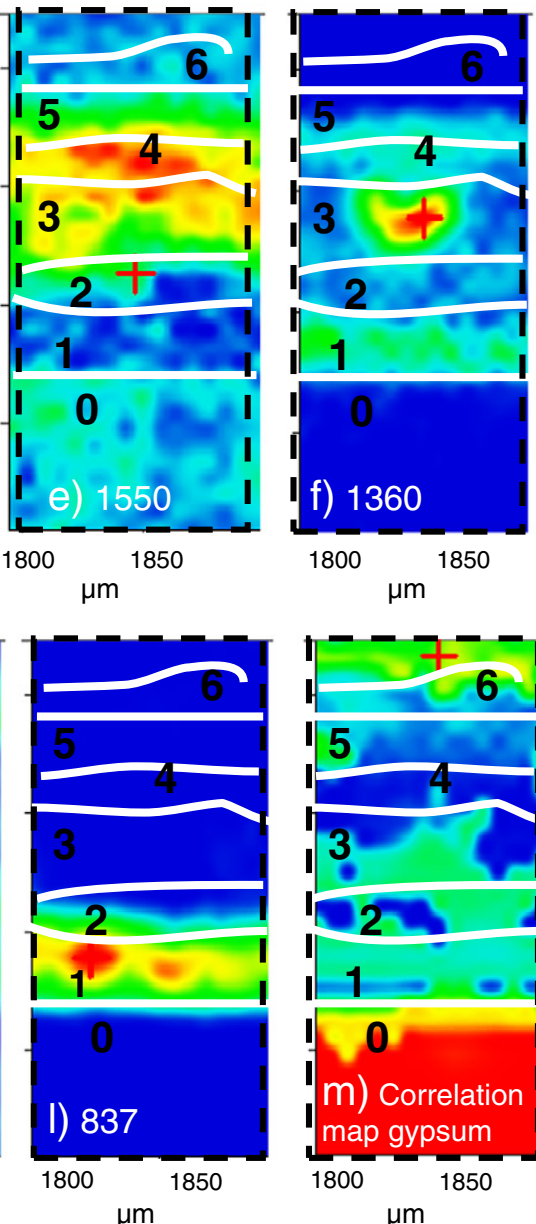

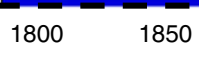
$\mu \mathrm{m}$

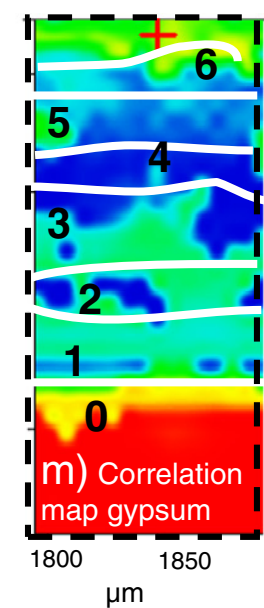

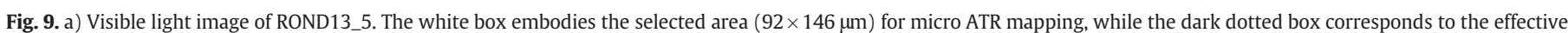
analyzed area after the crystal contact; b) FTIR false colours plots of the different painting components (includes the bands employed for the mapping, referring to Table 2). 

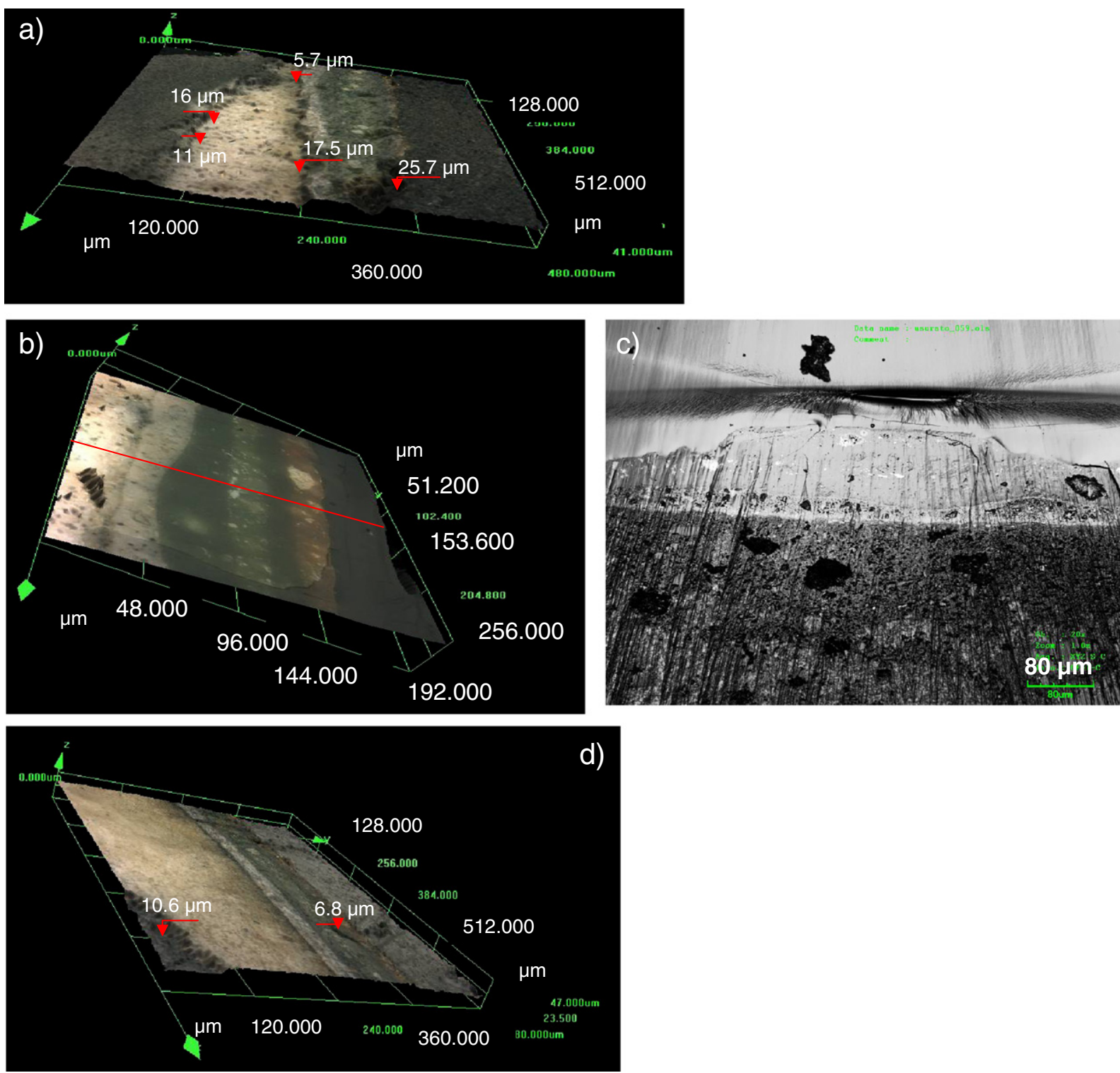

Fig. 10. Confocal microscopy images of a)3D ROND13_3, b) 3D ROND13_4, c) 2D ROND13_4, d) 3D ROND13_5.

Layers 2-4 were characterized by the presence of both acid and ester spectral bands at respectively 1710 and $1740 \mathrm{~cm}^{-1}$. In ROND13_3 and ROND13_4 the acid and the ester bands could be clearly distinguished in the green layers 2-4 (Fig. 11a,b). In ROND13_5 the two bands could still be detected, but the resolution was worse compared to the other samples (Fig. 11c). Spectral features ascribable to copper acetate or resinate pigments (bands at around 1605 and $1445 \mathrm{~cm}^{-1}$ ) could be detected only as shoulders in all the three cross sections, although their presence was confirmed by micro Raman analysis (data not reported). All the sample spectra collected from layers 2-4 (see Fig. 11) were dominated by bands ranging from $1547-1555 \mathrm{~cm}^{-1}$ to $1404-1410 \mathrm{~cm}^{-1}$. It is known that verdigris can be a mixture of different copper complexes. Recent studies, reproducing old recipes for the synthesis of verdigris, identified three different types of copper acetate derivatives [24]. The reported ATR-FTIR spectrum assigned to $\mathrm{Cu}\left(\mathrm{CH}_{3} \mathrm{COO}\right)_{2} \cdot 3 \mathrm{Cu}$ $(\mathrm{OH})_{2} \cdot 2 \mathrm{H}_{2} \mathrm{O}$ shows absorption at 1542 and $1405 \mathrm{~cm}^{-1}$, which may fit into the same spectral range of the bands detected on all the six ROND13 samples. However, this is also the spectral region of lead acetate, a degradation product which might have generated from the interaction between the original verdigris and a lead white pigment.
A specific research, focusing on this particular matter, is currently going on.

Interesting bands ranging from 1350 to $1250 \mathrm{~cm}^{-1}$ were collected from the green layers. In ROND13_4 two defined bands at 1376 and $1351 \mathrm{~cm}^{-1}$ were present together, with a well defined band at $3569 \mathrm{~cm}^{-1}$ (Fig. 11b). These three bands seemed to be related to each other because, when mapped, they gave rise to the same distribution. Unfortunately their assignment is still unclear. Due to the change of the set area, it was not possible to establish whether that unknown compound was present in layer 2 or 3. In ROND13_3 the band at 1375 and 1349 were present only as shoulder (Fig. 11a), while the $\mathrm{OH}$ absorption was completely absent. It is known that, in ATR, bands at lower wavenumbers are enhanced compared with what observed in transmission, and those present at higher wavenumbers are less intense or absent [18]. The lack of this band may thus indicate a worst contact with the crystal. These absorptions were not detected in ROND13_5. This may suggest that probably this compound was absent, or present in a lower amount. Only in ROND13_5 bands at 1360 and $1320 \mathrm{~cm}^{-1}$ were detected in layers 2-3 (Fig. 11c), which may suggest the presence of copper oxalate, even though the region around $1600 \mathrm{~cm}^{-1}$ was not enough defined. 

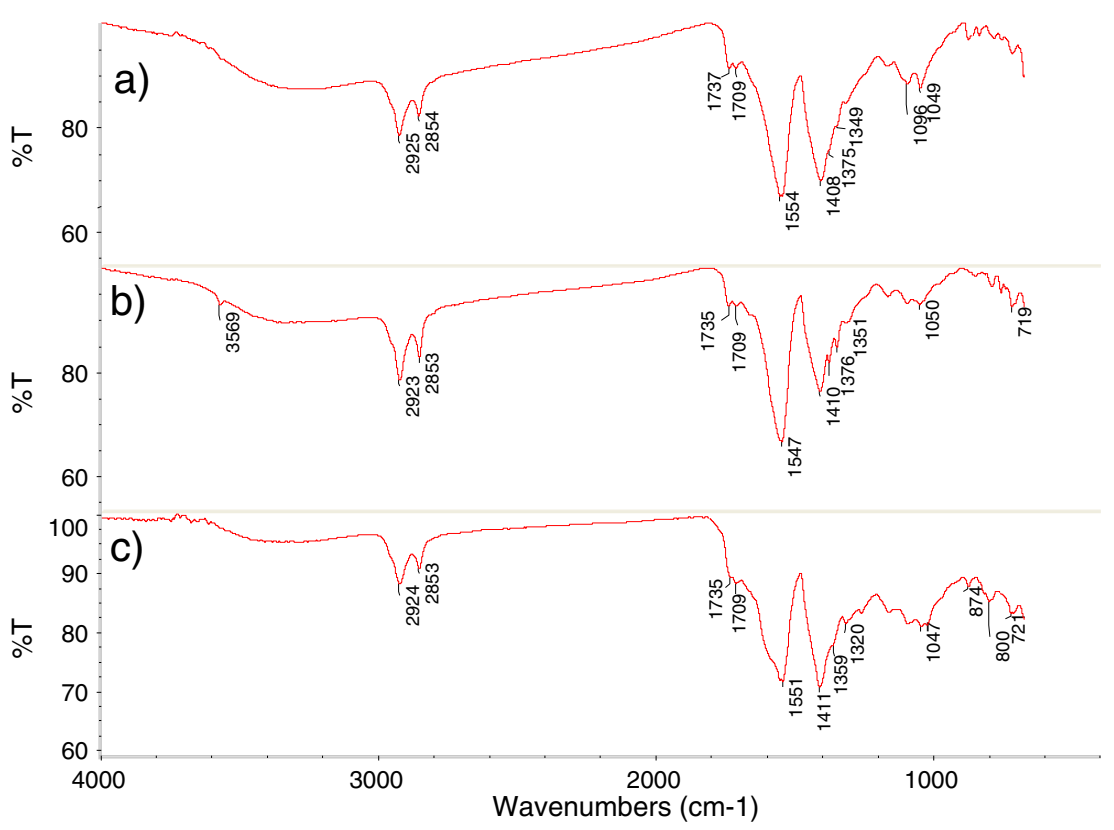

Fig. 11. Spectra from the green layers obtained in FTIR microscopy in ATR mode in sample a) ROND13_3; b) ROND13_4; c) ROND13_5.

The $\mathrm{KBr}$ embedded sample was indeed not well resolved in this region and in some points particularly noisy. Comparing the spectra of the different points in the map and focusing on the $1600-1800 \mathrm{~cm}^{-1}$ range, the spectral quality seemed to decrease from the first point of the map (ground preparation) to the end of the set area (varnish), due to the presence of hygroscopic $\mathrm{KBr}$ particles as observed by ESEM-EDX. Layer 5 (the gilding mordant) was as well identified in ROND13_3 and ROND13_5 (Figs. 5e, 9h) as a mixture of an earth type pigment mixed with an oily binder. It was not present in ROND13_4, where the analyzed area resulted different from the set one. This sample probably moved both horizontally and vertically in an area where the stratigraphy was not complete. In this case the movement was probably caused mainly by the ridges created by ion milling. Furthermore, the 12-hour treatment allowed only a small area of the sample to be polished, producing a step between the unpolished and the polished region, which made it difficult for the ATR crystal to make contact. The situation could be improved by simply extending the ion milling treatment to polish a larger area.

As for the oily component, spectra registered in layer 5 showed that the acidic component was predominant both in ROND13_3 and in ROND13_5. In ROND13_5 the resolution of the acidic and the ester bands was comparable to what observed in layers $2-4$, while in ROND13_3 spectra were particularly noisy. It can be noticed that, while the spectral resolution in ROND13_5 decreased with the passing of time due to the hygroscopic behaviour of the $\mathrm{KBr}$ particles present on the surface, in ROND13_3 spectra were probably affected by the irregular morphology of the different points, as shown by the image acquired by confocal microscopy (Fig. 10a).

Oxalates along with silicates were found in the final uppermost layer of varnish, visible only in ROND13_5, the sample embedded in $\mathrm{KBr}$ (Fig. 8f). The origin of oxalates can be addressed to deposition or deterioration of the organic protective coating responsible for the UV fluorescence that was seen under optical microscopy (Fig. 8f). While the oxalate map was well defined by integrating the $\mathrm{C}-\mathrm{O}$ stretching mode at $1320 \mathrm{~cm}^{-1}$, the signal of the fluorescent protective was too weak and noisy to be identified and mapped.

\section{Conclusions}

This research allowed to evaluate the effect of different embedding and polishing methods on the performance of micro FTIR in
ATR mode. As already known, it was confirmed that wet polishing may cause dissolution or erosion of water sensitive materials and should be avoided because the non homogeneous areas created on the sample may be hardly analysed in FTIR microscopy in ATR mode. On the other hand, it has been demonstrated that dry polishing may cause the contamination by some abrasive papers.

Infiltration into the outer layers and smearing of the organic embedding medium over the cross-section during polishing may also occur, causing significant contamination of the sample and affecting the IR investigation, especially when strongly absorbing infrared organic resins are used. This effect may be reduced if a pre-treatment with cyclododecane is performed and the cross section is obtained by cutting or if the resin embedded cross section is polished by ion milling. The use of IR transparent salts, such as $\mathrm{KBr}$, prevents interferences when performing FTIR analyses. Moreover, only the two samples embedded with this inorganic salt allowed the visualization of the final external protective layer. However, ESEM-EDX analyses evidenced the presence of $\mathrm{KBr}$ particles on the surface in the dry polished sections and their hygroscopic behaviour may lead to noisy spectra in the region around $1600 \mathrm{~cm}^{-1}$ along with a certain attenuation of the recorded infrared signal. Moreover, this effect increases with the passing of time, with the first spectra of a map with a better spectral quality than the last ones. The sample treated with cyclododecane and cut represents a good compromise for the identification of organic substances. However, the cutting procedure performed in wet conditions causes the partial dissolution of the water sensitive materials (like gypsum and glue of the preparation), while the presence of surface irregularities due to the lack of final polishing procedures may locally lead to less sensitive and more poorly defined spectra. Some tests are currently being carried out in order to favour cyclododecane infiltration as consolidant thus acting as barrier not only from the organic embedding resin but also from the solubilisation of water sensitive materials during the cutting procedure. The argon ion milled sample provided intense and well resolved spectra. Even though ridges were created on the surface that could cause the sample to move, extending the time of polishing could allow a larger smooth surface to be obtained. However, argon ion milling is extremely time consuming and expensive, and for this reason it cannot be proposed as an ordinary methodology. It is advisable, though, when analyzing important and rare samples. 


\section{Acknowledgment}

Part of this research was funded by the Italian Ministry of Education, University and Research (MIUR) through the PRIN 2008 project (prot. 2008ZRSHHB) and by the European project "CHARISMA" FP7 INFRASTRUCTURE n. 228330. We wish to thank JEOL for performing the Argon Ion Milling on ROND13_4 sample.

\section{References}

[1] J. Allen, Paint sample presentation for Fourier transform infrared microscopy, Vib. Spectrosc. 3 (1992) 217-237.

[2] R. Mazzeo, E. Joseph, S. Prati, A. Millemaggi, Attenuated Total Reflection-Fourier transform infrared microspectroscopic mapping for the characterisation of paint cross-sections, Anal. Chim. Acta 599 (2007) 107-117.

[3] J. Van der Weerd, R.M.A. Heeren, J.J. Boon, Preparation methods and accessories for the infrared spectroscopic analysis of multi-layer paint films, Stud. Conserv. 29 (2004) 193-210.

[4] J.S. Tsang, R.H. Cunningham, Some improvements in the study of cross sections, J. Am. Inst. Conserv. 30 (1991) 163-177.

[5] M. Cotte, E. Checroun, V. Mazel, A. Solé, P. Richardin, Y. Taniguchi, P. Walter, J. Susini, Combination of FTIR and X-RAYS Synchrotron-Based Micro-Imaging Techniques for the Study of Ancient Paintings. A Practical Point of View, E-Preserv. Sci. 6 (2009) 1-9.

[6] J.P. Echard, L. Bertrand, A. von Bohlen, A.S. Le Hô, C. Paris, L. Bellot-Gurlet, B. Soulier, A. Lattuati-Derieux, S. Thao, L. Robinet, B. Lavédrine, S. Vaiedelich, The nature of the extraordinary finish of Stradivari's instruments, Angew. Chem. Int. Ed. 49 (2010) 197-201.

[7] M. Derrick, L. Souza, T. Kieslick, H. Florsheim, D. Stulik, Embedding paint crosssection samples in polyester resins: problems and solutions, J. Am. Inst. Conserv. 33 (1994) 227-245.

[8] C.M. de Fonjaudran, A. Nevin, F. Piqué, S. Cather, Stratigraphic analysis of organic materials in wall painting samples using micro-FTIR attenuated total reflectance and a novel sample preparation technique, Anal. Bioanal. Chem. 392 (2008) 77-86.

[9] J. Pilc, R. White, The application of FTIR-microscopy to the analysis of paint binders in easel paintings, Natl. Gallery Tech. Bull. 16 (1995) 73-84.

[10] S. Prati, E. Joseph, G. Sciutto, R. Mazzeo, New advances in the application of FTIR microscopy and spectroscopy for the characterization of artistic materials, Acc. Chem. Res. 43 (2010) 792-801.
[11] A. Langley, A. Burnstock, The analysis of layered paint samples from modern paintings using FTIR microscopy, in: J. Bridgland (Ed.), Proceeding of the 12th Triennial Meeting of the ICOM Committee for Conservation, Lyon 29th August-3 rd September 1999, Vol. I, James \& James (Sciences Publishers) Ltd., London, 1999, pp. 234-241.

[12] J.J. Boon, S. Asahina, Surface preparation of cross sections of traditional and modern paint using the Argon ion milling polishing CP system, Microsc. Microanal. 12 (2006) 1322-1323.

[13] A. van Loon, K. Keune, J.J. Boon, Identifying and localizing proteinaceous compounds in paint samples using reflexion infrared spectroscopic techniques, Art'05 - 8th International Conference On The Non Destructive Investigations And Microanalysis For The Diagnostic And Conservation Of The Cultural And Environmental Heritage, Lecce, 2005.

[14] E. Jäegers, Volatile binding media: Useful tools for conservation, Br. Mus. Occas. Pap. 37 (1999) 42135 (1999).

[15] V. Muros, J. Hirx, The use of cyclododecane as a temporary barrier for watersensitive ink on archaeological ceramics during desalination, J. Am. Inst. Conserv. 43 (2004) 75-89.

[16] M. Spring, C. Ricci, D.A. Peggie, S.G. Kazarian, ATR-FTIR imaging for the analysis of organic materials in paint cross sections: case studies on paint samples from the National Gallery, London, Anal. Bioanal. Chem. 392 (2008) 37-45.

[17] E. Joseph, S. Prati, G. Sciutto, M. Ioele, P. Santopadre, R. Mazzeo, Performance evaluation of mapping and linear imaging FTIR microspectroscopy for the characterisation of paint cross sections, Anal. Bioanal. Chem. 396 (2010) 899-910.

[18] J.A. Reffner, P.A. Martoglio, Uniting Microscopy and Spectroscopy. Practical Guide to Infrared Microspectroscopy, in: H.J. Humecki (Ed.), Marcel Dekker, New York, 1995, pp. 41-84.

[19] L.L. Lewis, A.J. Sommer, Attenuated total internal reflection infrared mapping microspectroscopy of soft materials, Appl. Spectrosc. 54 (2000) 324-330.

[20] J.E. Katon, Infrared microspectroscopy. A review of fundamentals and applications, Micron 27 (1996) 303-314.

[21] R. Bhargava, I.W. Levin, Spectrochemical Analysis Using Infrared Multichannel Detector, Blackwell publishing Ltd., Oxford, 2005.

[22] M. Römer, K. Zecho, J. Meinhardt, Slope cutting with a broad $\mathrm{Ar}$ ion beam for SEM investigations - studies of artefacts on porous, inhomogeneous and temperaturesensitive materials', MC2009, in: G. Kothleitner, M. Leisch (Eds.), Instrumentation and Methodology, Vol. 1, 2009, Verlag der Tu, Graz.

[23] D.V. Thompson, Il libro dell'arte, the craftsman's handbook of Cennino d'Andrea Cennini, Dover, New York, 1954.

[24] M. San Andres, J.M. de la Roja, V.G. Baonzab, N. Sanchoa, Verdigris pigment: a mixture of compounds. Input from Raman spectroscopy, J. Raman Spectrosc. 41 (2010) 1478-1486. 